\title{
DIFICULDADE DE APRENDIZAGEM: PRINCIPAIS ABORDAGENS TERAPÊUTICAS DISCUTIDAS EM ARTIGOS PUBLICADOS NAS PRINCIPAIS REVISTAS INDEXADAS NO LILACS DE FONOAUDIOLOGIA NO PERÍODO DE 2001 A 2005
}

\author{
Learning difficulties: main therapeutic approaches \\ in articles published in the main speech therapy journals \\ from 2001 to 2005 - indexed on Lilacs
}

Tereza Cristina Ferraz de Lima ${ }^{(1)}$, Ana Cláudia Rodrigues Gonçalves Pessoa (2)

\begin{abstract}
RESUMO
Objetivo: verificar artigos referentes às dificuldades de aprendizagem, tendo como objetivos específicos classificar o tipo de artigo, além de analisar a abordagem e a nomenclatura utilizada para identificação dos problemas de aprendizagem, pelos autores dos mesmos, nas revistas de 2001 a 2005 com indexação no LILACS. Métodos: foram selecionados artigos com base nas seguintes palavras chaves: dificuldade de aprendizagem, distúrbio de aprendizagem, dificuldade de leitura e/ou escrita, dislexia, disortografia e disgrafia. Resultados: o tipo de artigo mais publicado foi artigo original, seguido de estudo de caso. A principal nomenclatura utilizada pelos autores foram distúrbios ou dificuldades de aprendizagem, e a abordagem mais citada foi aquela que contempla a consciência fonológica. Verificou-se 207 artigos em linguagem, sendo 22 referentes ao tema desse trabalho. Conclusão: existe uma escassez de publicação de trabalhos na área de linguagem, principalmente ao tema relacionado à dificuldade de aprendizagem que talvez seja explicado pela falta de consenso entre os autores, levando tanto a uma dificuldade diagnóstica como terapêutica.
\end{abstract}

DESCRITORES: Transtornos de Aprendizagem; Linguagem; Leitura; Escrita Manual; Dislexia

\section{INTRODUÇÃO}

A dificuldade de leitura e escrita, também conhecida como dificuldade de aprendizagem, é uma das patologias fonoaudiológicas que mais gera dúvidas em relação ao diagnóstico e processo terapêutico principalmente por não existir um consenso entre os autores sobre a caracterização de tal patologia ${ }^{1}$.

Lidar com o insucesso escolar constitui-se em tarefa complexa e desafiadora para a qual não se tem ainda uma resposta acabada, o que aponta para a necessidade de buscar alternativas que

\footnotetext{
(1) Fonoaudióloga; Clínica Tereza Ferraz de Lima.

(2) Fonoaudióloga; Professora da Faculdade Integrada do Recife; Hospital Barão de Lucena em Recife - PE; Doutora em Educação pela Universidade Federal de Pernambuco.
}

possam minimizar tal situação ${ }^{2}$. Apesar disso, a identificação de dificuldades de aprendizagem apresentadas por escolares vem crescendo e, conseqüentemente novas concepções sobre o processo de ensino/aprendizagem surgem, valorizando variáveis internas, como por exemplo, as expectativas e emoções tanto daqueles que ensinam como dos aprendizes ${ }^{3,4}$.

Os anos iniciais de escolarização parecem ser cruciais, no que diz respeito a aprender a ler. As experiências de aquisições positivas de leitura, nos primeiros dois anos e meio de escolarização formal, são favoráveis para uma visão positiva do aprendiz sobre si mesmo ${ }^{5}$.

Em relação à escrita, a criança cria hipóteses e as testa. Durante esse processo de construção passa por níveis distintos que variam do pré-silábico ao alfabético. Ao atingir esse último nível, a criança já consegue dominar o sistema notacional ${ }^{6}$. 
O maior problema da criança acontece na fase pósalfabética, em que ela percebe que não existe uma relação direta entre os sons e as letras, tendo que descobrir a norma ortográfica ${ }^{7}$.

A norma ortográfica do português é composta por regras regulares e irregulares. No caso das regulares pode-se inferir a forma correta porque existe um "princípio gerativo", uma regra que se aplica a várias palavras da língua, podendo ser compreendida pelo aprendiz, diferentemente das regras irregulares que dependem do processo de memorização, nesse último caso, o uso de uma letra é justificado apenas pela tradição de uso ou pela origem (etimologia) da palavra ${ }^{7}$. Para alguns autores, um aspecto importante para a aprendizagem da escrita é o desenvolvimento das habilidades metalingüísticas que correspondem a manifestações explícitas de uma consciência funcional das regras de organização ou uso da linguagem ${ }^{8}$. Dentre essas habilidades a metafonológica é aquela que gera maior controvérsia. Para alguns autores, o desenvolvimento de tais habilidades é imprescindível para a aquisição da escrita, para outros, a aquisição da escrita facilita o domínio de tais habilidades. Na verdade, as tarefas utilizadas nos estudos envolvem diferentes graus de habilidades cognitivas ${ }^{9-13}$. O tipo de tarefa selecionada para realizações de pesquisas científicas pode apontar resultados distintos, dependendo dos processos cognitivos envolvidos ${ }^{14}$. Além disso, muitas tarefas de habilidades metafonológicas envolvem alguma forma de processamento de memória ${ }^{15}$.

É consenso entre os autores que não existe uma hierarquia estrita entre as tarefas de habilidades fonológicas, porém, a consciência fonêmica seria a última a ser dominada pelo aprendiz, podendo ser facilitada pelo aprendizado da leitura e pela memória ${ }^{16}$. Essa hierarquia parece se estender para outras línguas, estudo desenvolvido com crianças gregas também comprova que a consciência de sílabas precede a consciência de fonemas ${ }^{17}$. É possível perceber ainda que crianças pré-escolares podem apresentar sensibilidade fonêmica quando ainda não são capazes de manipular explicitamente os fonemas da palavra falada ${ }^{18}$.

Para algumas crianças a aprendizagem da escrita pode não ocorrer de forma satisfatória gerando alterações de aprendizagem. Em relação a essas alterações existe controvérsia no que diz respeito à nomenclatura. As diferentes nomenclaturas utilizadas dependem da visão do estudioso que observa as alterações de leitura e escrita que, com suas abordagens teóricas diversificadas, influenciam o modo como o problema é definido. Apesar das diferentes teorias, a maioria desses profissionais concorda que nem todas as crianças que apre- sentam dificuldades para aprender a ler podem ser consideradas portadoras de distúrbios de leitura e escrita ${ }^{13}$.

Dentre as várias nomenclaturas e definições para os problemas específicos de aprendizagem pode-se citar: disortografia e disgrafia para problemas específicos de escrita, dislexia para problemas de leitura, distúrbio da aprendizagem, dificuldade específica de aprendizagem e dificuldade de leitura ${ }^{1,13,19,20}$.

Uma dificuldade específica de leitura é definida pela ocorrência de problemas significativos no reconhecimento de palavras em crianças que apresentam inteligência média, fluência na língua materna, nenhum déficit sensorial primário ou problemas emocionais ${ }^{21}$. Porém, essa mesma definição é utilizada por outros autores para as dislexias de desenvolvimento ${ }^{22}$. Outros autores defendem que a dificuldade de aprendizagem é uma condição de vulnerabilidade psicossocial ${ }^{23}$. Por esta razão, é comum que as crianças com tais problemas apresentem problemas emocionais ou déficits em habilidades sociais ${ }^{20,24}$, que durante a adolescência podem levar à evasão escolar ${ }^{25}$. Muitas vezes, podem ser desenvolvidos sentimentos de baixa auto-estima e inferioridade, como também déficits em habilidades sociais e problemas emocionais ou de comportamento ${ }^{26}$.

Diante do exposto, os fonoaudiólogos podem ter idéias claras sobre o problema de aprendizagem para orientar a equipe pedagógica e a família, e elaborar um plano terapêutico adequado quando necessário.

O que se busca neste trabalho é realizar um levantamento de artigos referentes às dificuldades de aprendizagem, na tentativa de classificar o tipo de artigo, de analisar a abordagem e a nomenclatura utilizadas para identificação dos problemas de aprendizagem pelos autores nas revistas de 2001 a 2005 com indexação no LILACS.

\section{MÉTODOS}

Para a construção desse estudo foram utilizadas obras literárias didáticas e realizou-se uma busca por artigos científicos, utilizando a base de dados LILACS. Foram encontradas quatro revistas específicas de Fonoaudiologia sendo elas, Pro-Fono, Fono Atual, Distúrbios da Comunicação e Revista CEFAC.

A partir deste levantamento foram selecionadas as revistas de 2001 a 2005 e verificaram-se quantos artigos foram publicados nas mesmas na área de linguagem, dentre os artigos selecionados foram observados especificamente aqueles referentes às questões de aprendizagem. 


\section{PROTOCOLO DE REGISTRO DOS ARTIGOS}

01. Identificação do Artigo:

Título:

Autores:

Ano:

Referência:

Número utilizado para o autor:

Letra utilizada para a revista:

02. Tipo de artigo:
( ) Revisão de literatura
( ) Estudo de caso
( ) Artigos originais
( ) Resumos de tese e/ou dissertação
( ) Outros:

03. Principal discussão no artigo:
( ) Diagnóstico
( ) Tratamento
( ) Nomenclatura
( ) Visão de especialistas
( ) Outros:

04. Enfoque principal:
( ) Leitura
( ) Escrita
( ) Leitura e escrita
( ) Outros:

05. Abordagem utilizada no artigo:
( ) Discursiva
( ) Biológico
( ) Social
( ) Educacional
( ) Outras:

06. Tipo de tratamento sugerido:
( ) Não se aplica.
( ) Psicológico
( ) Fonoaudiológico
( ) Médico
( ) Outros:

07. Descrever a abordagem fonoaudiológica, se for o caso.

08. Nomenclatura utilizada pelo autor para as alterações de aprendizagem e sua definição.

Figura 1 - Protocolo utilizado no registro dos artigos

Foram considerados como artigos referentes à aprendizagem os que abordavam os temas: dificuldade de aprendizagem, distúrbio de aprendizagem, dislexia, disortografia, disgrafia, alterações de leitura, alterações de escrita e alterações de leitura e escrita.

Os artigos selecionados foram lidos e registrados os principais dados em um protocolo estruturado para esse fim. Tal protocolo trata-se de um resumo das principais características do artigo, como por exemplo, tipo de artigo, principais discussões apresentadas pelos autores, nomenclaturas utilizadas e definições dadas a tais nomenclaturas (Figura 1).

A análise foi feita através de cálculos descritivos simples em percentuais e apresentados em tabela para facilitação da discussão.

Por questões éticas esse trabalho não citou o nome dos autores dos artigos e nos resultados também não será citado o nome das revistas. Tendo suas identificações sendo realizadas através de números (1 a 18) para os autores e letras (A, B,C,D) para os tipos das revistas.

\section{RESULTADOS}

Foram encontradas nas revistas pesquisadas 207 artigos na área de linguagem correspondendo a $26 \%$ do total de artigos em fonoaudiologia, destes apenas 22 estavam relacionados às dificuldades de aprendizagem, os quais foram analisados neste estudo. Vale ressaltar que os artigos em linguagem compreendem o segundo maior número do total de publicações, sendo a área com maior publicação a de audiologia (39\%) (Tabela 1).

$\mathrm{Na}$ Tabela 2 é possível observar a distribuição dos artigos que se referem às dificuldades de aprendizagem e que são de interesse desse estudo segundo o ano de publicação e o tipo da revista. Verifica-se que existe uma periodicidade maior de publicação do tema na revista do tipo $D$, com um total de 8 artigos o que corresponde a $36 \%$ do número total de publicações.

Os dados apontam que o tipo de artigo mais publicado refere-se a artigos originais $(n=12)(n \%$ $54,54)$, seguido de estudo de caso $(n=6)(n \% 27,27)$, revisão de literatura $(n=2)(n \% 9,0)$ e outros $(n=2)$ $(n \% 9,0)($ Tabela 3). Houve uma tendência dos artigos em abordar as dificuldades de aprendizagem dentro de uma visão biológica $(n=13) \quad(n \% 59,09)$ (Tabela 4). Conseqüentemente os problemas de aprendizagem são atribuídos exclusivamente à criança.

Nos artigos analisados foi percebida uma variedade de nomenclatura, sendo a mais comum "distúrbio ou dificuldade de aprendizagem" $(n=7)$ $(n \% 31,8)$ (Tabela 5). Os autores desses artigos quando utilizam tal nomenclatura se referem aos problemas da linguagem escrita, ocorrendo uma grande variedade nos conceitos. Esses problemas aparecem relacionados a atraso de linguagem e 
Tabela 1 - Distribuição da quantidade de artigos encontrados nas revistas pesquisadas referente ao ano de 2001 a 2005 separados pelas áreas específicas de Fonoaudiologia

\begin{tabular}{lcccccc}
\hline Especialidade/Revista & A & B & C & D & Valor absoluto & Total \% \\
\hline Linguagem & 31 & 54 & 69 & 53 & 207 & $26 \%$ \\
Motricidade oral & 97 & 26 & 36 & 25 & 184 & $23 \%$ \\
Audiologia & 49 & 66 & 147 & 47 & 309 & $39 \%$ \\
Voz & 21 & 13 & 49 & 12 & 95 & $12 \%$ \\
\hline
\end{tabular}

Tabela 2 - Distribuição dos artigos publicados nas revistas pesquisadas apresentadas de acordo com o ano de publicação cujo enfoque foi o tema de abordagem nesse estudo

\begin{tabular}{lcccc}
\hline $\begin{array}{l}\text { Ano/Revista } \\
\mathbf{N = 2 2}\end{array}$ & A & B & C & D \\
\hline 2001 & 1 & 2 & 0 & 1 \\
2002 & 3 & 0 & 1 & 1 \\
2003 & 1 & 0 & 2 & 3 \\
2004 & 0 & 1 & 1 & 1 \\
2005 & 0 & 2 & 0 & 2 \\
\hline Total & $23 \%$ & $23 \%$ & $18 \%$ & $36 \%$ \\
\hline
\end{tabular}

Tabela 3 - Dados dos tipos de artigos encontrados nas revistas pesquisadas

\begin{tabular}{lcccc}
\hline $\begin{array}{l}\text { Revista } \\
\mathbf{N = 2 2}\end{array}$ & $\begin{array}{c}\text { Revisão de } \\
\text { literatura }\end{array}$ & Estudo de caso & Artigos originais & Outros \\
\hline A & 0 & 0 & 5 & 0 \\
B & 1 & 1 & 3 & 0 \\
C & 0 & 2 & 0 & 2 \\
D & 1 & 3 & 4 & 0 \\
\hline Total & $9 \%$ & $27.27 \%$ & $54.54 \%$ & $9 \%$ \\
\hline
\end{tabular}

Tabela 4 - Freqüência das abordagens utilizadas nos artigos das revistas

\begin{tabular}{lccccc}
\hline Revista & Biológico & Social & Educacional & Discursiva & Outros \\
$\mathbf{N = 2 2}$ & 4 & 0 & 1 & 0 & 0 \\
\hline A & 3 & 0 & 1 & 1 & 0 \\
B & 2 & 0 & 0 & 1 & 1 \\
C & 4 & 1 & 2 & 1 & 0 \\
D & $59.09 \%$ & $4.54 \%$ & $18.18 \%$ & $13.63 \%$ & 4.54 \\
\hline Total & & & & \\
\hline
\end{tabular}

trocas surdo/sonoras, alterações no processamento auditivo central e consciência fonológica, questões educacionais, falta de estimulação da família, e até respiração mista.

É possível observar ainda que o termo dislexia aparece como sinônimo de distúrbio específico de leitura $(n=2)(n \% 9,1)$ e como transtornos ou dificul- dades de leitura e escrita $(n=3)(n \% 13,7)$. Quando utilizado o termo transtornos ou dificuldades de leitura e escrita observa-se que o artigo analisado de número 12 afirma que esse distúrbio não apresenta uma etiologia capaz de explicá-lo, enquanto que o artigo de número 2 considera esse distúrbio decorrente de processamento fonológico. 
Tabela 5 - Distribuição das nomenclaturas encontradas e a quantidade dos textos

\begin{tabular}{lcc}
\hline Nomenclatura & $\begin{array}{c}\text { Quantidade de textos } \\
\mathbf{N = 2 2}\end{array}$ & $\mathbf{n} \%$ \\
\hline Distúrbio específico de leitura ou dislexia & 2 & $9.1 \%$ \\
Distúrbio ou dificuldade de aprendizagem & 7 & $31.8 \%$ \\
Dislexia & 2 & $9.1 \%$ \\
Transtornos ou dificuldades de leitura e escrita ou dislexia & 3 & $13.7 \%$ \\
Dificuldade ou distúrbio de leitura e escrita & 4 & $18.2 \%$ \\
Problemas de escrita & 1 & $4.5 \%$ \\
Dificuldades ortográficas & 2 & $9.1 \%$ \\
Disortografia & 1 & $4.5 \%$ \\
\hline
\end{tabular}

Tabela 6 - Freqüência da discussão principal dos artigos nas revistas

\begin{tabular}{lcccc}
\hline Revista & Diagnóstico & Tratamento & $\begin{array}{c}\text { Visão de } \\
\text { especialistas }\end{array}$ & Outros \\
$\mathbf{N = 2 2}$ & 2 & 0 & 0 & 3 \\
\hline A & 2 & 2 & 1 & 0 \\
B & 1 & 2 & 1 & 0 \\
C & 5 & 0 & 0 & 3 \\
D & $45.45 \%$ & $18.18 \%$ & $9 \%$ & $27.27 \%$ \\
\hline Total & &
\end{tabular}

O termo dificuldade ou distúrbio de leitura e escrita aparece em 4 artigos (n\%18,2), sendo que 3 desses artigos apresentam como etiologia desse quadro alterações no processamento auditivo e um artigo aponta como causa os problemas da consciência fonológica.

Finalmente na Tabela 6, observa-se que a discussão mais freqüente ocorre em torno do diagnóstico das dificuldades de aprendizagem $(n=10)$ ( $\mathrm{n} \% 45,45)$, seguido de temas mais gerais, como por exemplo, caracterização do problema, classificado como outros $(n=6)(n \% 27,27)$, tratamento $(n=4)(n \%$ $18,18)$ e visão de especialistas $(n=2)(n \% 9,0)$.

\section{DISCUSSÃO}

Em relação à quantidade de publicações separadas pelas áreas específicas da Fonoaudiologia, observa-se uma escassez sobre dificuldade de aprendizagem, o que talvez possa refletir em uma atuação Fonoaudiológica com pouca base teórica. Tal fato sugere a necessidade de um maior investimento em estudos na área para consolidar a prática clínica do fonoaudiólogo.

A busca constante pelo conhecimento em relação aos problemas de leitura e escrita é crucial, porque existem problemas que estão relacionados aos aspectos da política educacional, ou ainda questões que se referem ao próprio processo de desenvolvimento do aprendiz, o que pode dificultar a realização de um diagnóstico diferencial. Dessa forma, é importante que o fonoaudiólogo conheça detalhadamente todo o processo de construção de escrita ${ }^{6}$ até o nível pós-alfabético ${ }^{12}$, entendendo todas as dificuldades naturais do processo de apreensão da norma ortográfica ${ }^{7}$.

A maior parte dos artigos coletados foi do tipo artigo original, seguido de estudo de caso. Talvez, isso possa ser explicado pelo fato dos estudiosos na área não apresentarem um consenso em relação às alterações, levando os fonoaudiólogos a buscar compreensão para tais problemas através de trabalhos de pesquisa ou estudo de caso ${ }^{1}$.

Chamou atenção nos resultados a ênfase dada à questão biológica como causa dos problemas de aprendizagem na maioria dos artigos analisados, ou seja, a princípio, o problema de leitura/escrita estaria relacionado a questões de saúde da criança. Esse tipo de visão não é incorreto dependendo da linha de estudo que o profissional prioriza na sua investigação, pois as questões etiológicas e de nomenclatura estão ligadas à própria formação do pesquisador ${ }^{13}$.

Embora vários cientistas sejam unânimes na existência de múltiplas causas das dificuldades de aprendizagem, não existe consenso quanto às cau- 
sas que são primárias e às que são subseqüentes, referindo à existência de fatores biológicos, destacando os fatores genéticos, pré, peri e pós-natais; fatores neurológicos e neuropsicológicos; e fatores sociais, como privação e classes sociais ${ }^{1}$.

A maior freqüência de discussão dos artigos está mais relacionada a questões de diagnóstico. A importância de se chegar a um diagnóstico satisfatório é imprescindível para que o fonoaudiólogo identifique se a dificuldade apresentada pela criança está ou não relacionada a questões de competência do fonoaudiólogo. Por esta razão, a troca com profissionais de áreas afins é imprescindível, visto que o diagnóstico das alterações de leitura e escrita é feito por uma equipe multidisciplinar ${ }^{19,26}$.

A realização de um diagnóstico criterioso é importante para evitar equívocos que possam estigmatizar o aluno em seu processo de aprendizagem e dificultar a superação de obstáculos ${ }^{27}$. A rotulação pode levar a criança a "acreditar" na sua dificuldade evitando, assim, a superação da mesma ${ }^{20}$.

Quando se observa nos resultados algumas controvérsias no momento de definir os problemas relacionados à leitura e escrita, subtende-se que elas tenham sido geradas a partir das diferentes concepções desses problemas. Nos primeiros relatos literários sobre o assunto, as dificuldades de leitura e escrita aparecem vinculadas à presença de alterações orgânicas. Com o surgimento da teoria lingüística de Chomsky passa-se a levar em conta não somente as condições internas de aprendizagem, mas também a valorizar as condições externas, representadas pelo campo de estímulos recebidos e que também poderiam interferir positivamente ou negativamente na aprendizagem ${ }^{28}$.

Quando se observa nos resultados algumas controvérsias no momento de definir os problemas relacionados à leitura e escrita, subtende-se que elas tenham sido geradas a partir das diferentes concepções desses problemas. Nos primeiros relatos literários sobre o assunto, as dificuldades de leitura e escrita aparecem vinculadas à presença de alterações orgânicas. Com o surgimento da teoria lingüística de Chomsky passa-se a levar em conta não somente as condições internas de aprendizagem, mas também a valorizar as condições externas, representadas pelo campo de estímulos recebidos e que também poderiam interferir positivamente ou negativamente na aprendizagem ${ }^{28}$.

Verificando as definições dadas pelos autores dos artigos analisados percebeu-se uma preferência pelo uso do termo dislexia, o que parece supor que o diagnóstico é dado a qualquer dificuldade que se verifique no aprendizado da leitura. $\mathrm{Na}$ verdade, essa definição corresponde a um diagnóstico referente à dislexia de evolução, que considera as alterações durante o aprendizado da leitura (e escrita) não importando qual a sua causa ${ }^{29}$.

Alguns artigos também apresentam como sinônimo as terminologias "dificuldade de aprendizagem" e "distúrbio de aprendizagem", porém existem diferenças entre elas. A primeira refere-se a um déficit específico da atividade escolar, enquanto a segunda refere-se a uma disfunção intrínseca à criança, em geral neurológica ou neuropsicológica ${ }^{30}$. É possível afirmar que quando a dificuldade de leitura é causada por um dano neurológico, pode ser diagnosticada uma dislexia adquirida ${ }^{31}$.

Conforme exposto é provável que as diferenças de conceituação possam gerar dificuldades de diagnóstico e, conseqüentemente, problemas nas propostas terapêuticas adequadas.

\section{CONCLUSÃO}

Analisando a publicação na área de linguagem nas revistas Pró-Fono, Fono Atual, CEFAC e Distúrbios da Comunicação verificou-se uma predominância de artigos originais. Percebeu-se ainda que existiu uma tendência dos autores analisados em discutir as dificuldades de aprendizagem dentro de uma abordagem biológica, levando a conceber o problema como intrínseco ao indivíduo.

Quanto à nomenclatura utilizada para as dificuldades de aprendizagem, não existiu um consenso podendo ser verificadas para uma mesma caracterização do problema nomenclaturas distintas.

Diante do exposto pode-se sugerir que ainda são escassas as publicações na área de linguagem em relação às dificuldades específicas de aprendizagem. 


\begin{abstract}
Purpose: to check articles regarding learning difficulties, with the specific objectives of classifying the type of article as well as analyzing the approach and nomenclature that the authors used in the identification of learning problems in publications from 2001 to 2005 - indexed on LILACS. Methods: survey of articles taking into consideration the following key words: learning difficulty, learning disability, reading and/or writing difficulty, dyslexia, dysortography and dysgraphy. Results: original article was the most published, followed by case study. The main nomenclature that the authors used were "learning disability" or "learning difficulty", and the most cited approach was the one addressing phonological awareness. We observed 207 articles on language, 22 of these articles referred to the subject of the present study. Conclusion: the studies published on the field of language are scarce, especially regarding subjects related to learning difficulties. Perhaps this scarcity can be explained by a lack of consensus among authors, leading to both diagnostic as well as therapeutic difficulties.
\end{abstract}

KEYWORDS: Learning Disorders; Language; Reading; Handwriting; Dyslexia

\section{REFERÊNCIAS}

1. Fonseca V. Introdução às dificuldades de aprendizagem. Porto Alegre: Artes Médicas; 1995. 388 p.

2. Okano CB, Loureiro SR, Linhares MBM, Marturano EM. Crianças com dificuldades escolares atendidas em programa de suporte psicopedagógico na escola: avaliação do autoconceito. Psicol Reflex Crit. 2004; 17(1):121-8.

3. Boruchovitch E. As variáveis psicológicas e o processo de aprendizagem: uma contribuição para a psicologia escolar. Psicol Teor Pesq. 1994; 10(1):129-39.

4. Chapman JW, Cullen JL, Boersma FJ, Maguire TO. Affective variables and school achievement: a study of possible causal influences. Can J Behav Sci. 1981; 3:181-92.

5. Chapman JW, Tunmer WE. A longitudinal study of beginning reading achievement and reading selfconcept. Br J Educ Psychol. 1997; 67(3):279-91.

6. Ferreiro $E$, Teberosky A. Psicogênese da língua escrita. Porto Alegre: Artmed; 1986. 300 p.

7. Morais AG. Ortografia: ensinar e aprender. São Paulo: Ática; 1998. 128 p.

8. Gombert JE. Atividades metalingüísticas e aprendizagem da leitura. In: Maluf MR. Metalinguagem e aquisição da escrita: contribuições da pesquisa para a prática da alfabetização. São Paulo: Casa do Psicólogo; 2003. p. 19-63.

9. Morais AG, Lima NC. Análise fonológica e compreensão da escrita alfabética: um estudo com crianças de escola pública. Anais do Simpósio Latino-Americano de Psicologia do Desenvolvimento; 1989.

10. Maluf MR, Barrera SD. Consciência fonológica e linguagem escrita em pré-escolares. Psicol Reflex Crit. 1997; 10(1):125-45.
11. Brayant $P$, Bradley $L$. Problemas da leitura na criança. Porto Alegre: Artes Médicas; 1997. 137 p.

12. Nunes T, Buarque L, Bryant P. Dificuldades na aprendizagem da leitura: teoria e prática. 4. ed. São Paulo: Cortez; 1992.112 p.

13. Santos MTM, Navas ALGP. Distúrbios de leitura e escrita: teoria e prática. São Paulo: Manole; 2002. $389 \mathrm{p}$.

14. Treiman R. Introdution to special issue on spelling. Read Writ Interdiscipl J. 1997; 9:315-9.

15. Cormier P, Dea S. Distinctive patterns of relationship of phonological awareness and working memory with reading development. Read Writ Interdiscipl J. 1997; 9:193-206.

16. Freitas GCM. Sobre a consciência fonológica. In: Lamprecht RR. Aquisição fonológica do português: perfil do desenvolvimento e subsídios para terapia. Porto Alegre: Artmed; 2004. p. 177-92.

17. Aidinis A, Nunes T. The role of different levels of phonological awareness in the development of reading and spelling in greek. Read Writ Interdiscipl J. 2001; 14:145-77.

18. Bowey JA. Synthatic awareness and verbal performance form preschool to fifth grade. J Psycholinguist Re. 1994; 15(4):285-308.

19. Leão FC. Avaliação em voz, fala e linguagem. In: Jakubovicz R. Avaliação em voz, fala e linguagem. São Paulo: Revinter; 2004. p. 163-79.

20. Carneiro GRS, Martinelli SC, Sisto FF. Autoconceito e dificuldades de aprendizagem na escrita. Psicol Reflex Crit. 2003; 16(3):427-34.

21. Wise BW, Ring J, Olson RK. Training phonological awareness with and without explicit attention to articulation. J Exp Child Psychol. 1999; 72(4):271-304.

22. Pinheiro AMV. Dificuldades específicas de leitura: a identificação de déficits cognitivos e a abor- 
dagem do processamento de informação. Psicol Teor Pesq. 1995; 11(2):107-15.

23. Rutter M. Psychosocial resilience and protective mechanisms. Am J Orthopsychiatry. 1987; 57(3):316-31.

24. Kavale KA, Forness SR. Social skill deficits and learning disabilities: a meta-analysis. J Learn Disabil. 1996; 29(3):226-37.

25. Maughan B, Gray G, Rutter, M. Reading retardation and antisocial behaviour: a follow-up into employment. J Child Psychol Psychiatry. 1985; 26(5):741-58.

26. Santos LC, Marturano EM. Crianças com dificuldade de aprendizagem: um estudo de seguimento. Psicol Reflex Crit. 1999; 12(2):377-94.
27. Mollica MCM. O natural e o patológico na escrita. In.: Cadernos do IPUB. Rio de Janeiro: UFRJ; 2000. p. 17-25.

28. Tedesco MRM. Diagnóstico e terapia dos distúrbios do aprendizado da leitura e escrita. In: Lopes Filho O. Tratado de fonoaudiologia. São Paulo: Tecmedd; 1997. p. 647-62.

29. Santos CC. Dislexia específica de evolução. São Paulo: Sarvier; 1987. 207 p.

30. Capellini SA. Distúrbios de aprendizagem versus dislexia. In: Ferreira LP, Lopes DMB, Limongi SCO. Tratado de fonoaudiologia. São Paulo: Roca; 2004. p. 862-76.

31. Andrew WE. Leitura, escrita e dislexia: uma análise cognitiva. 2. ed. Porto Alegre: Artmed; 1995. 153 p.

Endereço para correspondência:

Rua Santa Catarina, 111 Qd. C BI. 3

Olinda - PE

CEP: $53230-251$

Tel: (81) 32713075

Fax: (81) 32245790

E-mail: aclaudiapessoa@fir.br claudia_pessoa@hotmail.com 